\title{
UNCONDITIONALLY CONVERGING MULTILINEAR OPERATORS
}

\author{
FERNANDO BOMBAL, MAITE FERNÁNDEZ-UNZUETA, AND IGNACIO VILLANUEVA
}

\begin{abstract}
We introduce a notion of unconditionally converging multilinear operator which allows to extend many of the results of the linear case to the multilinear case. We prove several characterizations of these multilinear operators (one of which seems to be new also in the linear case), which allow to considerably simplify the work with this kind of operators.
\end{abstract}

Keywords and phrases: Unconditionally converging, multilinear operators, weakly unconditionally Cauchy series.

Unconditionally converging operators were introduced by Pelczynski in [13] and since then, they have been extensively studied. In [9] a notion of unconditionally converging polynomial is introduced, but it seems to be too general, since the coincidence of this class with other classes of polynomials impose severe restrictions on the space (see [9, Theorem 10], e.g.). In [5] the author gives a new definition of unconditionally converging polynomial which allows to extend many of the results of unconditional converging operators (see [6]). In this note, we extend this definition to multilinear operators, proving some properties and characterizations, and studying the relationship existing with other definitions in the literature.

Let $E$ be a Banach space and $E^{*}$ its dual. We recall that a formal series $\sum_{n=1}^{\infty} x^{n} \subset E$ is said to be weakly unconditionally Cauchy if for every $x^{*} \in E^{*}$, the series $\sum_{n=1}^{\infty}\left|x^{*}\left(x^{n}\right)\right|$ converges (for equivalent definitions and basic properties of this kind of series we refer the reader to [4]).

Definition 1. Let $E_{1}, \ldots, E_{k}, X$ be Banach spaces. A multilinear operator $T \in$ $\mathcal{L}^{k}\left(E_{1}, \ldots, E_{k} ; X\right)$ is said to be unconditionally converging, and we will write $T \in \mathcal{L}_{u c}^{k}\left(E_{1}, \ldots, E_{k} ; X\right)$ if, for every weakly unconditionally Cauchy (w.u.C.) series $\sum x_{i}^{n} \subset E_{i}$, with $i \in\{1, \ldots, k\}$, the sequence $\left(T\left(s_{1}^{m}, \ldots, s_{k}^{m}\right)\right)_{m}$ converges in norm, where $s_{i}^{m}=\sum_{n=1}^{m} x_{i}^{n}$.

Analogously, a polynomial $P \in \mathcal{P}\left({ }^{k} E ; X\right)$ is said to be unconditionally converging, and we will write $P \in \mathcal{P}_{u c}\left({ }^{k} E ; X\right)$ if, for every weakly unconditionally converging series $\sum x^{n} \subset E$, the sequence $\left(P\left(s^{m}\right)\right)_{m}$ converges in norm, where $s^{m}=\sum_{n=1}^{m} x^{n}([5])$.

Recall that there is a canonical isomorphism between $\mathcal{L}^{k}\left(E_{1}, \ldots, E_{k} ; X\right)$ and $\mathcal{L}\left(E_{1} \hat{\otimes} \cdots \hat{\otimes} E_{k} ; X\right)$, where $E_{1} \hat{\otimes} \cdots \hat{\otimes} E_{k}$ denotes projective tensor product. A continuous multilinear map $T \in \mathcal{L}^{k}\left(E_{1}, \ldots, E_{k} ; X\right)$ is said to be compact (resp. weakly compact) if the corresponding linear operator on the

1991 Mathematics Subject Classification. 46B28.

Key words and phrases. unconditionally converging,multilinear operators.

First and third authors are partially supported by DGICYT grant PB97-0240.

Second author is partially supported by Conacyt grant I 29875-E. 
tensor product is compact (resp. weakly compact); equivalently, if $T$ maps the product of the corresponding unit balls into a relatively compact (resp., weakly compact) subset of $X$.

Using the polarization formula, (see [12, pg. 6]) it is easy to check that a polynomial is unconditionally converging if and only if so is its associated symmetric multilinear generator.

In the linear case, a direct consequence of the Orlicz-Pettis theorem is that the unconditionally converging operators are precisely those which transform w.u.C series into weakly convergent ones. We shall show that this result extends to the multilinear case. The proof is a suitable modification of the corresponding result given in [6] for polynomials. We shall need the following extension of [3, Lemma 7.4]:

Lemma 2. Let $E_{1}, \ldots, E_{k}$ be Banach spaces, of which all but at most one have the Dunford-Pettis property. Let $\left\{x_{i}^{n}\right\}_{n=1}^{\infty},\left\{y_{i}^{n}\right\}_{n=1}^{\infty}$ be weakly Cauchy sequences in $E_{i},(1 \leq i \leq k)$ such that $\left\{x_{i}^{n}-y_{i}^{n}\right\}_{n=1}^{\infty}$ is weakly null $\left.(1 \leq i \leq k)\right)$. Then $\left\{x_{1}^{n} \otimes \cdots \otimes x_{k}^{n}-y_{1}^{n} \otimes \cdots \otimes y_{k}^{n}\right\}_{n=1}^{\infty}$ is a weak null sequence in $E_{1} \hat{\otimes} \cdots \hat{\otimes} E_{k}$.

Proof. We have

$$
\begin{gathered}
x_{1}^{n} \otimes \cdots \otimes x_{k}^{n}-y_{1}^{n} \otimes \cdots \otimes y_{k}^{n}=\left(x_{1}^{n}-y_{1}^{n}\right) \otimes x_{2}^{n} \otimes \cdots \otimes x_{k}^{n}+ \\
+y_{1}^{n} \otimes\left(x_{2}^{n}-y_{2}^{n}\right) \otimes \cdots \otimes x_{k}^{n}+\ldots+y_{1}^{n} \otimes \cdots \otimes y_{k-1}^{n} \otimes\left(x_{k}^{n}-y_{k}^{n}\right) .
\end{gathered}
$$

Every member of the right side has $k-1$ factors which are weak Cauchy sequences, and the other factor is a weak null sequence. Hence, by the arguments of $[3$, Lemma 7.4] each summand converges weakly to zero in the tensor product.

Theorem 3. Let $E_{1}, \ldots, E_{k}, X$ be Banach spaces. A multilinear operator $T \in$ $\mathcal{L}^{k}\left(E_{1}, \ldots, E_{;} X\right)$ is unconditionally converging if and only if, for every weakly unconditionally Cauchy series $\sum x_{i}^{n} \subset E_{i}$, with $i \in\{1, \ldots, k\}$, the sequence $\left(T\left(\sum_{n=1}^{m} x_{1}^{n}\right.\right.$, $\left.\left.\ldots, \sum_{n=1}^{m} x_{k}^{n}\right)\right)_{m}$ converges weakly in $X$.

Proof. Suppose $T$ satisfy the weaker hypothesis, but it is not unconditionally converging. Then, there are w.u.C. series $\sum x_{i}^{n}$ in $E_{i}(1 \leq i \leq k), \epsilon>0$ and a pair of subsequences $\left(n_{j}\right),\left(m_{j}\right)$ with $n_{j}>m_{j}$, such that

$$
\left\|T\left(s_{1}^{n_{j}}, \ldots, s_{k}^{n_{j}}\right)-T\left(s_{1}^{m_{j}}, \ldots, s_{k}^{m_{j}}\right)\right\| \geq \epsilon,
$$

where $s_{i}^{m}=\sum_{n=1}^{m} x_{i}^{n}$. Let

$$
S: c_{o} \times \cdots \times c_{o} \rightarrow E_{1} \times \cdots \times E_{k}
$$

be the continuous $k$-linear map defined by

$$
\begin{gathered}
S\left(\sum a_{1}^{n} e_{n}, \ldots \sum a_{k}^{n} e_{n}\right)=\left(S_{1}\left(\sum a_{1}^{n} e_{n}\right), \ldots S_{k}\left(\sum a_{k}^{n} e_{n}\right)\right):= \\
:=\left(\sum a_{1}^{n} x_{1}^{n}, \ldots \sum a_{k}^{n} x_{k}^{n}\right),
\end{gathered}
$$

where $\left(e_{n}\right)$ is the usual basis in $c_{o}$, and put $V:=T \circ S$. With this notation, we have

$$
\begin{aligned}
& T\left(s_{1}^{n_{j}}, \ldots, s_{k}^{n_{j}}\right)-T\left(s_{1}^{m_{j}}, \ldots, s_{k}^{m_{j}}\right)=T\left(s_{1}^{n_{j}}-s_{1}^{m_{j}}, s_{2}^{n_{j}}, \ldots, s_{k}^{n_{j}}\right)+ \\
+ & T\left(s_{1}^{m j}, s_{2}^{n_{j}}-s_{2}^{m_{j}}, s_{3}^{n_{j}}, \ldots, s_{k}^{n_{j}}\right)+\ldots+T\left(s_{1}^{m_{j}}, \ldots, s_{k-1}^{m_{j}}, s_{k}^{n_{j}}-s_{k}^{m_{j}}\right) \\
= & V\left(z_{j}-w_{j}, z_{j}, \ldots, z_{j}\right)+\ldots+V\left(w_{j}, \ldots, w_{j}, z_{j}-w_{j}\right)
\end{aligned}
$$


with $z_{j}:=\sum_{k=1}^{n_{j}} e_{k}, w_{j}:=\sum_{k=1}^{m_{j}} e_{k} \in c_{o}$. Then $\left(z_{j}\right),\left(w_{j}\right)$ are weakly Cauchy sequences, and $\left(z_{j}-w_{j}\right)$ converges weakly to zero. Hence, by Lemma 2 for instance, each member of the right side of the above sum converges weakly to zero. If we were able to prove that they converge in norm, we should get a contradiction to $(*)$ and the proof would be over. But Pelczynski showed in [14] that every weakly compact multilinear operator on a product of spaces with the Dunford-Pettis property (like $c_{o}$ ), transforms weakly Cauchy sequences into a norm convergent one. Therefore, it suffices to prove the following

Claim: $V$ is weakly compact.

In fact, let $\left(v_{1}^{n}\right), \ldots\left(v_{k}^{n}\right)$ be $k$ sequences in the unit ball of $c_{o}$. Passing to some subsequences if necessary, we can suppose that all of them are weakly Cauchy. Property $(u)$ of $c_{o}$ (see $\left.[11,1 . c .1]\right)$ guarantees the existence of w.u.C. series $\sum w_{i}^{n}, 1 \leq$ $i \leq k$, such that the sequences $\left(\sigma_{i}^{m}-v_{i}^{m}\right)$ are weakly null for $i=1, \ldots, k$ (where $\left.\sigma_{i}^{m}=\sum_{n=1}^{m} w_{i}^{n}\right)$. By Lemma $2\left(\sigma_{1}^{m} \otimes \cdots \otimes \sigma_{k}^{m}-v_{1}^{m} \otimes \cdots \otimes v_{k}^{m}\right)$ is weakly null and so

$$
V\left(\sigma_{1}^{m}, \ldots, \sigma_{k}^{m}\right)-V\left(v_{1}^{m}, \ldots, v_{k}^{m}\right) \stackrel{w}{\rightarrow} 0 .
$$

But, by hypothesis, $V\left(\sigma_{1}^{m}, \ldots, \sigma_{k}^{m}\right)=T\left(\sum_{n=1}^{m} S_{1}\left(w_{1}^{n}\right), \ldots \sum_{n=1}^{m} S_{k}\left(w_{k}^{n}\right)\right)$ converges weakly to some $x \in X$ (since $\sum S_{i}\left(w_{i}^{n}\right)$ are w.u.C. series in $\left.E_{i}\right)$, and thus $V\left(v_{1}^{m}, \ldots, v_{k}^{m}\right)$ also converges weakly to $x$, which proves the claim.

We remark that the above proof can be repeated word for word to show that whenever $E_{1}, \ldots E_{k}$ are Banach spaces with the Dunford-Pettis property, the property $(\mathrm{u})$ of Pelczynski and without copies of $\ell_{1}$, every $k$ - linear unconditionally converging operator on $E_{1} \times \ldots \times E_{k}$ is weakly compact. When all the spaces coincide with $c_{o}$, we can say more:

Corollary 4. Every $k$-linear unconditionally converging operator on $c_{o} \times \ldots \times c_{O}$ is compact.

Proof. As we have mentioned before, every $T \in \mathcal{L}_{u c}^{k}\left(c_{o}, \ldots, c_{o} ; X\right)$ is weakly compact. By induction on $k$, using the facts that every operator from $c_{o}$ into $c_{o}^{*}\left(\approx \ell_{1}\right)$ is compact and that $c_{o}^{*}$ has the approximation property, it can be easily proved that the dual of $c_{o} \hat{\otimes} \cdots \hat{\otimes} c_{o}$ is the injective tensor product of $k$ spaces $\ell_{1}$, and hence a Schur space, since this property is preserved by taking injective tensor products (see [15, Proposition 1.3], e.g.). If $\tilde{T} \in \mathcal{L}\left(c_{o} \hat{\otimes} \cdots \hat{\otimes} c_{o} ; X\right)$ is the operator corresponding to $T$, it is weakly compact and, by Gantmacher's theorem, so is its transpose $\tilde{T}^{*}$. But the range of this operator is a Schur space. Therefore, $\tilde{T}^{*}$ and hence $T$ are compact, by Schauder's theorem.

The above result is also true if instead of $c_{o}$ we take any $C(\Omega)$ space, with $\Omega$ a compact scattered Hausdorff topological space. The proof is the same, once we know that every $T \in \mathcal{L}_{u c}^{k}\left(C\left(\Omega_{1}\right), \ldots, C\left(\Omega_{k}\right) ; X\right)$ is weakly compact, which was proved in [10].

Also embedded in the proof of Theorem 3 is the result that the sequence $\left\{T\left(\sum_{n=1}^{m} x_{1}^{n}\right.\right.$, $\left.\left.\ldots, \sum_{n=1}^{m} x_{k}^{n}\right)\right\}_{m}$ is weakly Cauchy for every $T \in \mathcal{L}^{k}\left(E_{1}, \ldots, E_{k} ; X\right)$ and w.u.C. series $\sum x_{i}^{n} \subset E_{i}, 1 \leq i \leq k$. In fact, with the above notation,

$$
T\left(\sum_{n=1}^{m} x_{1}^{n}, \ldots, \sum_{n=1}^{m} x_{k}^{n}\right)=V\left(\sum_{n=1}^{m} e_{n}, \ldots \sum_{n=1}^{m} e_{n}\right)
$$


and $\left\{\sum_{n=1}^{m} e_{n} \otimes \cdots \otimes \sum_{n=1}^{m} e_{n}\right\}_{m}$ is weakly Cauchy by Lemma 2. An immediate consequence of this is the following corollary:

Corollary 5. i) Every weakly compact multilinear operator is unconditionally converging.

ii) If $X$ is weakly sequentially complete, every $T \in \mathcal{L}^{k}\left(E_{1}, \ldots, E_{n} ; X\right)$ is unconditionally converging.

Later we shall give more conditions under which every multilinear operator is unconditionally converging.

In [2, Theorem 2.3], a result about completely continuous multilinear operators is proved which turns out to be very useful in working with this kind of mapping. Now we shall prove an analogous result for unconditionally converging operators, which allows to mimic for this class of operators many results and techniques of completely continuous mappings, as can be seen for example in [10].

We will state first an auxiliary definition.

Definition 6. Let $E_{1}, \ldots, E_{k}, X$ be Banach spaces. A multilinear operator $T \in$ $\mathcal{L}^{k}\left(E_{1}, \ldots, E_{k} ; X\right)$ is said to be unconditionally continuous, if, for every weakly unconditionally Cauchy series $\sum_{n \in \mathbb{N}} x_{i}^{n} \subset E_{i}$ with $i=1, \ldots, k$ such that

$$
s_{i}^{n}=\sum_{m=1}^{n} x_{i}^{m} \stackrel{\omega}{\rightarrow} x_{i} \in E_{i}
$$

the following holds

$$
\lim _{n \rightarrow \infty}\left\|T\left(s_{1}^{n}, \ldots, s_{k}^{n}\right)-T\left(x_{1}, \ldots, x_{k}\right)\right\|=0 \quad .
$$

The above definition is clearly easier to handle than that of unconditionally converging operators. Our aim is to prove that both classes of operators coincide. In one direction the proof of this is based on the proof of [2, Lemma 2.4 and Theorem 2.3].

Theorem 7. Let $E_{1}, \ldots, E_{k}, X$ be Banach spaces and let $T \in \mathcal{L}^{k}\left(E_{1}, \ldots, E_{k} ; X\right)$. Then the following are equivalent:

i) $T$ is unconditionally converging.

ii) $T$ is such that if, for every $j=1, \ldots, k, \sum_{n=1}^{\infty} x_{j}^{n} \subset E_{j}$ is a weakly unconditionally Cauchy series and there exists $i \in\{1, \ldots, k\}$ such that $\sum_{n=1}^{\infty} x_{i}^{n}$ weakly converges to zero, then

$$
\lim _{m \rightarrow \infty}\left\|T\left(s_{1}^{m}, \ldots, s_{k}^{m}\right)\right\|=0 \text {, where } s_{j}^{m}=\sum_{n=1}^{m} x_{j}^{n} .
$$

iii) $T$ is unconditionally continuous.

Proof. i) $\Rightarrow$ ii): We will do the proof assuming without loss of generality that in ii), $i=1$. Let $T$ and $\sum_{n} x_{1}^{n}, \ldots, \sum_{n} x_{k}^{n}$ be as in the hypothesis. If the result is not true, then there exist an $\epsilon>0$ and an increasing sequence of indexes $J=(m(l))_{l}$ such that, for every $l \in \mathbb{N}$,

$$
\left\|T\left(s_{1}^{m(l)}, \ldots, s_{k}^{m(l)}\right)\right\|>\epsilon
$$

On the other hand, it is clear that, if we fix $m \in \mathbb{N}$, the operator $T_{m}=T_{s_{2}^{m}, \ldots, s_{k}^{m}} \in$ $\mathcal{L}\left(E_{1} ; X\right)$ defined as

$$
T_{m}(x)=T\left(x, s_{2}^{m}, \ldots, s_{k}^{m}\right)
$$


is unconditionally converging, and therefore there exists $n(m)>m$ such that for every $r \geq n(m)$,

$$
\left\|T_{m}\left(s_{1}^{r}\right)\right\|<\frac{\epsilon}{2} .
$$

We can assume that if $j \in J$, then $n(j) \in J$. Then, for every $j \in \mathbb{N}$,

$$
\left\|T\left(s_{1}^{m(l)}, \ldots, s_{k}^{m(l)}\right)-T\left(s_{1}^{n(m(l))}, s_{2}^{m(l)}, \ldots, s_{k}^{m(l)}\right)\right\|>\frac{\epsilon}{2}
$$

Let $p(0)=1, p(1)=n(m(1))$ and, if $l>1, p(l)=n(p(l-1))$. We then have that, for every $l \geq 1$,

$$
\left\|T\left(s_{1}^{p(l)}, \ldots, s_{k}^{p(l)}\right)-T\left(s_{1}^{p(l+1)}, s_{2}^{p(l)}, \ldots, s_{k}^{p(l)}\right)\right\|>\frac{\epsilon}{2} .
$$

Let us now define

$$
y_{1}^{l}= \begin{cases}\sum_{n=p(j)+1}^{p(j+1)} x_{1}^{n} & \text { if } l=2 j+1 \\ 0 & \text { if } l=2 j\end{cases}
$$

and for $i>1$

$$
y_{i}^{l}= \begin{cases}0 & \text { if } l=2 j+1 \\ \sum_{n=p(j-1)+1}^{p(j)} x_{i}^{n} & \text { if } l=2 j\end{cases}
$$

(with $p(r)=0$ if $r<0$.)

Then it is clear that $\sum_{n} y_{i}^{n} \subset E_{i}$ is a w.u.C. series for each $i \in\{1, \ldots, k\}$ and, if we call $\sigma_{i}^{m}=\sum_{n=1}^{m} y_{i}^{n}$, we get that

$$
\sigma_{1}^{2 j+1}=s_{1}^{p(j+1)}, \quad \sigma_{1}^{2 j}=\sigma_{1}^{2 j-1}=s_{1}^{p(j)}
$$

and, if $i>1$,

Therefore, for every $j \in \mathbb{N}$,

$$
\sigma_{i}^{2 j+1}=\sigma_{i}^{2 j}=s_{i}^{p(j)}
$$

$$
\begin{gathered}
\left\|T\left(\sigma_{1}^{2 j+1}, \ldots, \sigma_{k}^{2 j+1}\right)-T\left(\sigma_{1}^{2 j}, \ldots, \sigma_{k}^{2 j}\right)\right\|= \\
=\left\|T\left(s_{1}^{p(j+1)}, s_{2}^{p(j)}, \ldots, s_{k}^{p(j)}\right)-T\left(s_{1}^{p(j)}, s_{2}^{p(j)}, \ldots, s_{k}^{p(j)}\right)\right\|>\frac{\epsilon}{2}
\end{gathered}
$$

a contradiction to the fact that $T$ is unconditionally converging.

The proof that ii) $\Rightarrow$ iii) is easy considering that

$$
\begin{gathered}
\left\|T\left(s_{1}^{n}, \ldots, s_{k}^{n}\right)-T\left(x_{1}, \ldots, x_{k}\right)\right\| \leq\left\|T\left(s_{1}^{n}-x_{1}, s_{2}^{n}, \ldots, s_{k}^{n}\right)\right\|+ \\
+\left\|T\left(x_{1}, s_{2}^{n}-x_{2}, x_{3} \ldots, x_{k}\right)\right\|+\cdots+\left\|T\left(x_{1}, \ldots, x_{k-1}, s_{k}^{n}-x_{k}\right)\right\| .
\end{gathered}
$$

iii) $\Rightarrow$ ii): This proof is an adaptation of the proof of [2, Lemma 2.4]; we will do it by induction on $k$. If $k=1$ the result is clear. Let us suppose it true for $k-1$. Now we will consider the case of $k$-linear operators: if the result is false, for $1 \leq j \leq k$ there exist weakly unconditionally Cauchy series $\sum_{n=1}^{\infty} x_{j}^{n} \subset E_{j}$ where we suppose that $\sum_{n=1}^{\infty} x_{1}^{n}$ weakly converges to 0 , such that $\left(T\left(\sum_{n=1}^{m} x_{1}^{n}, \ldots, \sum_{n=1}^{m} x_{k}^{n}\right)_{m \in \mathbb{N}}\right.$ does not converge to 0 . Then there exist $\epsilon>0$ and an infinite subset $J \subset \mathbb{N}$ such that for every $j \in J$,

$$
\left\|T\left(\sum_{n=1}^{j} x_{1}^{n}, \ldots, \sum_{n=1}^{j} x_{k}^{n}\right)\right\|>\epsilon
$$


We will consider $J=\{j(1)<j(2)<\cdots<j(n)<\cdots\}$ Now let us fix $m \in \mathbb{N}$. The function

defined as

$$
T_{s_{k}^{j(m)}}: E_{1} \times \cdots \times E_{k-1} \mapsto X
$$

$$
T_{s_{1}^{j(m)}}\left(x_{1}, \ldots, x_{k-1}\right)=T\left(x_{1}, \ldots, x_{k-1}, \sum_{n=1}^{j(m)} x_{k}^{n}\right)
$$

is clearly unconditionally continuous. Then the induction hypothesis assures that there exist an index $p(m) \in \mathbb{N}$ such that

$$
\left\|T_{s_{1}^{j(m)}}\left(\sum_{n=1}^{l} x_{1}^{n}, \ldots, \sum_{n=1}^{l} x_{k-1}^{n}\right)\right\|<\epsilon / 2 \text { for every } l>p(m)
$$

Clearly we can choose $j(m)<p(m)<p(m+1)$ and $p(m) \in J$ for every $m \in \mathbb{N}$. In particular for every $m \in \mathbb{N}$ we get

$$
\begin{gathered}
\epsilon / 2 \leq\left\|T\left(\sum_{n=1}^{p(m)} x_{1}^{n}, \ldots, \sum_{n=1}^{p(m)} x_{k-1}^{n}, \sum_{n=1}^{p(m)} x_{k}^{n}\right)\right\|- \\
-\left\|T\left(\sum_{n=1}^{p(m)} x_{1}^{n}, \ldots, \sum_{n=1}^{p(m)} x_{k-1}^{n}, \sum_{n=1}^{j(m)} x_{k}^{n}\right)\right\|= \\
=\left\|T\left(\sum_{n=1}^{p(m)} x_{1}^{n}, \ldots, \sum_{n=1}^{p(m)} x_{k-1}^{n}, \sum_{n=1}^{p(m)} x_{k}^{n}-\sum_{n=1}^{j(m)} x_{k}^{n}\right)\right\|
\end{gathered}
$$

Let us consider now the series

$$
y_{i}^{m}=\sum_{n=p(m)+1}^{p(m+1)} x_{i}^{n} \text { for } i=1 \ldots k-1
$$

and

$$
y_{k}^{m}=\sum_{n=p(m)+1}^{p(m+1)} x_{k}^{n}-\sum_{n=j(m)+1}^{j(m+1)} x_{k}^{n} \text { for } i=k
$$

These $k$ series are weakly unconditionally Cauchy, they have the property that at least two of them (the first and the last) weakly sum to 0 and they verify that

$$
\left\|T\left(\sum_{n=1}^{m} y_{1}^{n}, \ldots, \sum_{n=1}^{m} y_{k}^{n}\right)\right\| \geq \epsilon / 2
$$

Repeating the reasoning we obtain k weakly unconditionally Cauchy series $\sum_{n=1}^{\infty} z_{i}^{n}$ such that all of them weakly sum to 0 and

$$
\left\|T\left(\sum_{n=1}^{j} z_{1}^{n}, \ldots, \sum_{n=1}^{j} z_{k}^{n}\right)\right\| \geq \epsilon /\left(2^{k-1}\right)
$$

which is a contradiction.

ii) $\Rightarrow$ i) Let $\sum x_{1}^{n} \subset E_{1}, \ldots, \sum x_{k}^{n} \subset E_{k}$ be weakly unconditionally Cauchy series and let $(p(r))_{r}$ and $(q(r))_{r}$ be two increasing sequences of indexes with $p(0)=q(0)=$ 0 . Then

$$
\begin{gathered}
\left\|T\left(s_{1}^{p(r)}, \ldots, s_{k}^{p(r)}\right)-T\left(s_{1}^{q(r)}, \ldots, s_{k}^{q(r)}\right)\right\|= \\
=\left\|T\left(s_{1}^{p(r)}-s_{1}^{q(r)}, s_{2}^{p(r)}, \ldots, s_{k}^{p(r)}\right)\right\|+\left\|T\left(s_{1}^{q(r)}, s_{2}^{p(r)}-s_{2}^{q(r)}, s_{3}^{p(r)}, \ldots, s_{k}^{p(r)}\right)\right\|+
\end{gathered}
$$




$$
+\cdots+\left\|T\left(s_{1}^{q(r)}, \ldots, s_{k-1}^{q(r)}, s_{k}^{p(r)}-s_{k}^{q(r)}\right)\right\|
$$

Let us see that each of these terms converges to zero when $r$ grows to infinity. We will prove it only for the second term, since the others are treated similarly. Let us consider the following series:

$$
\begin{gathered}
y_{1}^{m}=\sum_{n=p(m-1)+1}^{p(m)} x_{1}^{n}, \\
y_{2}^{m}=\sum_{n=p(m-1)+1}^{p(m)} x_{2}^{n}-\sum_{n=q(m-1)+1}^{q(m)} x_{2}^{n} \text { and } \\
y_{i}^{m}=\sum_{n=p(m-1)+1}^{p(m)} x_{i}^{n} \text { for } i=3, \ldots, k .
\end{gathered}
$$

Clearly, for every $i=1, \ldots, k, \sum y_{i}^{n}$ is a weakly unconditionally Cauchy series and besides $\sum y_{2}^{n}$ weakly converges to zero. Therefore the hypothesis proves that this term converges to zero, which finishes the proof.

We want to point out that this result seems to be new also in the linear case. The authors have verified that several proofs of results concerning unconditionally converging linear operators can be made much simpler by using it.

Of course the result implies the existence of weakly unconditionally Cauchy series that weakly converge to zero but do not converge weakly unconditionally. A simple and not new example of one such series is $\sum x_{n} \subset c_{0}$ where

$$
\begin{aligned}
& x_{1}=e_{1} \\
& x_{n}=e_{n}-e_{n-1} \quad \text { if } n>1 .
\end{aligned}
$$

As we mentioned at the beginning, a different definition of unconditionally converging polynomials which also extends the linear one was given in [9]. The natural extension to multilinear operators would be the following:

Definition 8. Let $E_{1}, \ldots, E_{k}, X$ be Banach spaces. A multilinear operator $T \in$ $\mathcal{L}^{k}\left(E_{1}, \ldots, E_{k} ; X\right)$ will be called weakly unconditionally converging, and we will write $T \in \mathcal{L}_{w u c}^{k}\left(E_{1}, \ldots, E_{k} ; X\right)$, if for every weakly unconditionally Cauchy series $\sum x_{i}^{n} \subset E_{i}$, with $i \in\{1, \ldots, k\}$, the series $\sum T\left(x_{1}^{n}, \ldots, x_{k}^{n}\right)$ converges in norm.

This definition gives rise to a strictly wider class, as the next two propositions show:

Proposition 9. Every unconditionally converging multilinear operator is weak unconditionally converging.

Proof. Let $E_{1}, \ldots, E_{k}, X$ and $\sum x_{i}^{n}$ be as in the above definition and let $T \in$ $\mathcal{L}_{u c}^{k}\left(E_{1}, \ldots, E_{k} ; X\right)$. The $k$-Rademacher generalized functions $\left\{s_{n}(t)\right\}_{n=1}^{\infty}$ ([1]) satisfy the following orthogonality properties:

$$
\int_{0}^{1} s_{i_{1}}(t) \cdots s_{i_{k}}(t) d t=\left\{\begin{array}{rr}
1 & \text { if } i_{1}=\ldots=1_{k} \\
0 & \text { otherwise }
\end{array}\right.
$$


Then

$$
\begin{aligned}
\sum_{n=1}^{m} T\left(x_{1}^{n}, \ldots, x_{k}^{n}\right) & =\int_{0}^{1} \sum_{i_{1} \cdots i_{k}=1}^{m} s_{i_{1}}(t) \cdots s_{i_{k}}(t) T\left(x_{1}^{i_{1}}, \ldots, x_{k}^{i_{k}}\right) d t \\
& =\int_{0}^{1} T\left(\sum_{i_{1}=1}^{m} s_{i_{1}}(t) x_{1}^{i_{1}}, \ldots, \sum_{i_{k}=1}^{m} s_{i_{k}}(t) x_{k}^{i_{k}}\right) d t .
\end{aligned}
$$

For every $t \in[0,1]$ the series $\sum s_{n}(t) x_{i}^{n}(1 \leq i \leq k)$ are weakly unconditionally Cauchy, and therefore the sequence $\left\{T\left(\sum_{i_{1}=1}^{m} s_{i_{1}}(t) x_{1}^{i_{1}}, \ldots, \sum_{i_{k}=1}^{m} s_{i_{k}}(t) x_{k}^{i_{k}}\right)\right\}$ converges in norm. Hence, $\sum_{n=1}^{\infty} T\left(x_{1}^{n}, \ldots, x_{k}^{n}\right)$ converges in norm, that is, $T$ is weakly unconditionally converging.

However, not every weak unconditionally converging operator is unconditionally converging. In fact, we have:

Proposition 10. Let $k>1$ and let $E_{1}, \ldots E_{k}$ be Banach spaces. The following assertions are equivalent:

i) $N o E_{1}, \ldots, E_{k}$ contains copies of $c_{o}$.

ii) $\mathcal{L}_{u c}^{k}\left(E_{1}, \ldots, E_{k} ; X\right)=\mathcal{L}^{k}\left(E_{1}, \ldots, E_{k} ; X\right)$, for every Banach space $X$.

iii) $\mathcal{L}_{u c}^{k}\left(E_{1}, \ldots, E_{k} ; X\right)=\mathcal{L}_{w u c}^{k}\left(E_{1}, \ldots, E_{k}: X\right)$, for every Banach space $X$.

Proof. If no $E_{i}$ contains copies of $c_{o}$, every w.u.C. series is converges in norm, and so $(i)$ implies $(i i)$.

Obviously, (ii) implies (iii).

Finally, let us prove that ( $i i i)$ implies $(i)$ : Suppose for instance that $E_{1}$ contains a copy of $c_{o}$ and let $\left(x_{1}^{n}\right)$ be a sequence equivalent to the usual $c_{o}$ basis. Choose norm one elements $x_{i}^{*} \in E_{i}^{*}(2 \leq i \leq k)$ and define

$$
T: E_{1} \times \cdots \times E_{k} \rightarrow E_{1}
$$

by the formula $T\left(z_{1}, \ldots, z_{k}\right):=x_{2}^{*}\left(z_{2}\right) \cdots x_{k}^{*}\left(z_{k}\right) z_{1}$. If $\sum y_{i}^{n} \subset E_{i}(1 \leq i \leq k)$ are w.u.C. series, we have

$$
\sum_{n=1}^{\infty}\left\|T\left(y_{1}^{n}, \ldots y_{k}^{n}\right)\right\| \leq\left(\sup _{n}\left\|y_{1}^{n}\right\| \cdots\left\|y_{k-1}^{n}\right\|\right)\left(\sum_{n=1}^{\infty}\left|x_{k}^{*}\left(y_{k}^{n}\right)\right|\right)<\infty
$$

which proves that $T$ is weakly unconditionally converging. However, if $z_{i} \in E_{i}$ ( $2 \leq$ $i \leq k)$ are chosen in such a way that $x_{i}^{*}\left(z_{i}\right)=1$ for $i=2, \ldots k$, then

$$
T\left(\sum_{n=1}^{m} x_{1}^{n}, z_{2}, \ldots, z_{k}\right)=\sum_{n=1}^{m} x_{1}^{n}
$$

which does not converge in norm. Therefore, $T$ is not unconditionally converging.

The following two propositions were told to us by J. Gutirrez, to whom thanks are given. Proposition 11 was proved in [7] under the stronger hypothesis that $X^{*}$ contains no copy of $\ell_{1}$. Proposition 12 is an application of Theorem 7 , and it refines [8, Theorem 3].

Proposition 11. If $X$ does not contain a copy of $c_{0}$, then for every Banach space $E_{1}, \ldots E_{k}$, every multilinear operator from $E_{1}, \ldots E_{k}$ into $X$ is unconditionally converging. 
Proof. Let $T \in \mathcal{L}^{k}\left(E_{1}, \ldots, E_{k} ; X\right)$ and let $\sum_{n} x_{1}^{n} \subset E_{1}, \ldots, \sum_{n} x_{k}^{n} \subset E_{k}$ be weakly unconditionally Cauchy series. Let us define $S \in \mathcal{L}^{k}\left(c_{0} ; E_{1} \times \cdots \times E_{k}\right)$ as in the proof of 3 , and put $Q:=T \circ S$.

From [8, Theorem 6] (or from [16]) it follows that $Q$ sends weakly Cauchy sequences in $E_{1} \times, \cdots, \times E_{k}$ into norm converging ones, and therefore it is unconditionally converging. So, the sequence

$$
Q\left(\sum_{n=1}^{m} e_{n}, \ldots, \sum_{n=1}^{m} e_{n}\right)=T\left(\sum_{n=1}^{m} x_{1}^{n}, \ldots, \sum_{n=1}^{m} x_{k}^{n}\right)
$$

converges, which implies that $T$ is unconditionally converging.

Proposition 12. Let $E_{1}, \ldots E_{k}$ and $X$ be Banach spaces such that $\mathcal{L}\left(E_{i} ; X\right)=$ $\mathcal{L}_{u c}\left(E_{i} ; X\right)$ for each $i \in\{1, \ldots, k\}$. Then it is also true that $\mathcal{L}^{k}\left(E_{1}, \ldots E_{k} ; X\right)=$ $\mathcal{L}_{u c}^{k}\left(E_{1}, \ldots E_{k} ; X\right)$.

Proof. Suppose first that one of the $E_{i}$ 's contains a complemented copy of $c_{0}$. Then $X$ does not contain a copy of $c_{0}$ and Proposition 11 gives us the result. Now, if none of the $E_{i}$ 's contains a complemented copy of $c_{0}$, then, by [8, Lemmas 1 and 2], we have that $\mathcal{L}\left(E_{i} ; c(X)\right)=\mathcal{L}_{u c}\left(E_{i} ; c(X)\right)$ (where $c(X)$ stands for the Banach space of all $X$-valued convergent sequences, endowed with the sup norm), and we proceed by induction on $k$. Suppose the result to be true for $k-1$ multilinear mappings, and let us consider $T \in \mathcal{L}^{k}\left(E_{1}, \ldots, E_{k} ; X\right)$ and weakly unconditionally Cauchy series $\sum_{n} x_{1}^{n} \subset E_{1}, \ldots, \sum_{n} x_{k}^{n} \subset E_{k}$ such that one of them, say the first one, weakly converges to zero. Let us define

$$
S: E_{1} \mapsto c(X)
$$

by

$$
S\left(x_{1}\right)=\left(T\left(x_{1}, s_{2}^{m}, \ldots, s_{k}^{m}\right)\right)_{m}
$$

where $s_{i}^{m}=\sum_{n=1}^{m} x_{i}^{n}$. From the induction hypothesis it is clear that $S$ is well defined. Since $S$ is an unconditionally converging operator, we get that

$$
\left\|T\left(s_{1}^{m}, \ldots, s_{k}^{m}\right)\right\| \leq\left\|S\left(s_{1}^{m}\right)\right\| \rightarrow 0
$$

which, by Theorem7, implies that $T$ is unconditionally converging.

\section{REFERENCES}

[1] R. M. Aron and J. Globevnik, Analytic functions on $c_{o}$, Revista Matemtica Univ. Complutense, 2 (1989), 27-33

[2] R. M. Aron, C. Hervés and M. Valdivia, Weakly continuous mappings on Banach spaces, J. Funct. Anal., 52, 1983, 189-204.

[3] T. K. Carne, B. Cole and T. W. Gamelin, A uniform Algebra of Analytic Functions on a Banach Space, Trans. Amer. Math. Soc., 314, 2 (1989), 639-659.

[4] J. Diestel, Sequences and Series in Banach Spaces, Graduate Texts in Math. 92, Springer, Berlin 1984.

[5] M. Fernández Unzueta, Unconditionally convergent polynomials in Banach spaces and related properties, Extracta Math. 12 (1997), 305-307.

[6] M. Fernández Unzueta, Unconditionally converging polynomials on Banach spaces, preprint.

[7] M. Fernndez Unzueta, Mdulos de Polinomios y Conjuntos Polinomiales en Espacios de Banach. Ph.D. Thesis. C.I.M.A.T., Guanajuato (Mxico), 1998.

[8] M. González and J. M. Gutiérrez, When every polynomial is unconditionally converging, Arch. Math. 63 (1994), 145-151.

[9] M. Gonzlez and J. Gutirrez, Unconditionally converging polynomials on Banach spaces, Math. Proc. Cambridge Philos. Soc., 117 (1995), 321-331.

[10] J. Gutirrez and I. Villanueva, Aron-Berner extensions and Banach space properties, Preprint. 
10 FERNANDO BOMBAL, MAITE FERNÁNDEZ-UNZUETA, AND IGNACIO VILLANUEVA

[11] J. Lindenstrauss and L. Tzafriri Classical Banach Spaces I, Springer-Verlag, Berlin-New York, 1977

[12] J. Mújica, Complex Analysis in Banach spaces, Math. Studies No. 120. North-Holland, 1986.

[13] A. Pelczynski, On Banach spaces on which every Unconditionally converging operator is weakly compact, Bull Acad. Pol. Sci. 10 (1962), 641-648.

[14] A. Pelczynki, On weakly compact polynomial operators on B-spaces with the Dunford-Pettis property, Bull. Acad. Pol. Sci., XI (1963), 371-378.

[15] W. Ruess and D. Werner, Structural properties of operator spaces, Acta Univ. Carol. 28 (1987), 127-136.

[16] I. Villanueva, Completely continuous multilinear operators on $C(K)$ spaces, to appear in Proc. Amer. Math. Soc.

Departamento de Anlisis Matemtico, Facultad de Matemticas, Universidad Complutense DE MADRID, MADRID 28040

E-mail address: bombal@eucmax.sim.ucm.es

C.I.M.A.T., A.P. 402, 36000, Guanajuato, Gto., Mxico

E-mail address: fernan@cimat.mx

Departamento de Anlisis Matemtico, Facultad de Matemticas, Universidad Complutense DE MADRID, MADRID 28040

E-mail address: ignacio_villanueva@mat.ucm.es 